\title{
Quasiclassical theory of superconducting multi-layers
}

\author{
Thomas Lück $^{\mathrm{a}, *}$, Ulrich Eckern ${ }^{\mathrm{a}}$, Andrei Shelankov ${ }^{\mathrm{b}}$ \\ a Institute of Physics, University of Augsburg, Augsburg 86135, Germany \\ ${ }^{\mathrm{b}}$ Department of Theoretical Physics, Umeå University, Umeå 901 87, Sweden
}

\begin{abstract}
We study the density of states of a thin normal metal layer in proximity to a bulk superconductor in the clean as well as in the dirty limit. We find that for increasing impurity scattering a mini gap is established in the normal metal. Furthermore, we consider a spin-active surface of the normal metal (e.g. due to an additional ferromagnetic layer) and observe a suppression of the mini gap.
\end{abstract}

Keywords: Superconductivity; Quasiclassical theory; Multi-layers

\section{Introduction}

We study a system consisting of a thin normal metal layer on top of a bulk superconductor as shown in Fig. 1 $\left(d_{n} \approx \xi_{0}, \xi_{0}:\right.$ superconducting coherence length). We calculate the density of states (DOS) in the normal metal layer using the quasiclassical theory of superconductivity. We generalize the earlier work of Belzig et al. [1] by considering arbitrary impurity concentrations and reduced transparency between the normal metal and the superconductor. Furthermore, we examine a spin-active surface which can be realized by an insulating ferromagnetic layer when in contact with the normal metal.

\section{Quasiclassical theory}

In thermal equilibrium, the quasiclassical Green's function $\hat{g}\left(E, \mathbf{p}_{F} ; \mathbf{r}\right)$ is determined by the Eilenberger equation and the normalization condition:

$\left[\hat{\tau}_{3} E+i \hat{\Delta}\left(\mathbf{p}_{F}, \mathbf{r}\right)+i \hat{\sigma}(\mathbf{r}), \hat{g}\right] i v_{F} \cdot \nabla \hat{g}=0$,

$\hat{g}^{2}=\hat{1}$.

\footnotetext{
*Corresponding author.

E-mail address: thomas.lueck@physik.uni-augsburg.de
} (T. Lück)
The hats denote the $4 \times 4$ matrix structure, which combines spin and particle-hole space $\left(\hat{\tau}_{i} / \hat{\sigma}_{i}\right.$ are the Pauli matrices in particle-hole/spin space); for simplicity we assume an identical spherical Fermi surface in the normal metal and the superconductor. The s-wave order parameter $\hat{\Delta}$ and the impurity self-energy $\hat{\sigma}$ (in Born approximation) must be determined self-consistently:

$$
\begin{aligned}
& \hat{\Delta}(\mathbf{r})=i \hat{\sigma}_{2} \pi T \mathcal{N}_{0} V \sum_{\left|E_{n}\right|<E_{c}} \frac{1}{2} \operatorname{Tr}_{\sigma}\left[i \hat{\sigma}_{2} \hat{g}_{\mathrm{s}}\left(i E_{n} ; \mathbf{r}\right)\right], \\
& \hat{\sigma}(E ; \mathbf{r})=\frac{1}{2 \tau} \hat{g}_{s}(E ; \mathbf{r}) .
\end{aligned}
$$

Here $\mathcal{N}_{0}$ is the DOS of the superconductor in the normal state, $V$ is the pairing interaction, and $\hat{g}_{s}$ is the swave part of the Green's function; $\operatorname{Tr}_{\sigma}$ is the trace in spin space. This approach allows us to study the cross-over from the clean $(1 / 2 \tau=0)$ to the dirty $\left(1 / 2 \tau>T_{c}\right)$ limit.

The quasiclassical theory is not directly applicable at interfaces and the Eilenberger equation must be supplemented by appropriate boundary conditions. The NS interface characterized by the transparency $\mathcal{T}_{0}$ is

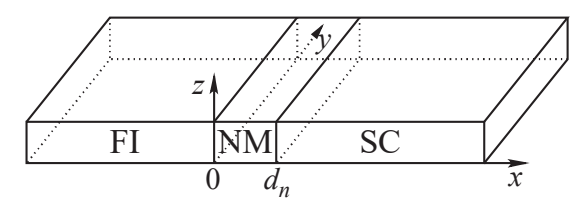

Fig. 1. F-N-S-structure studied in this work. 

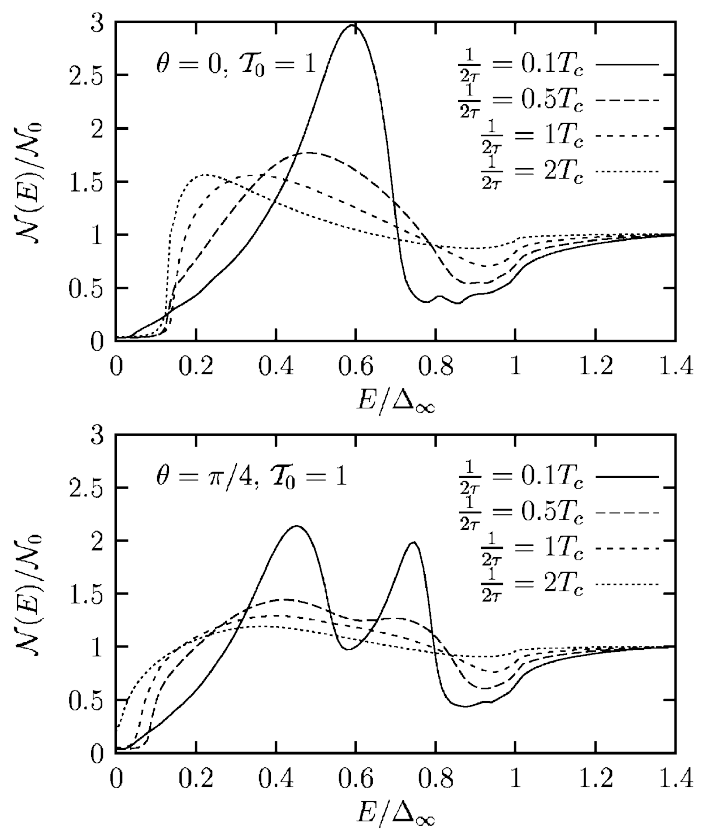

Fig. 2. For ideal transparency and large $1 / 2 \tau$ the mini gap, which is present for $\theta=0$, is suppressed for $\theta=\pi / 4$ with increasing impurity scattering; for small $1 / 2 \tau$ the splitting of the two spin channels for $\theta=\pi / 4$ can be observed.

incorporated with the help of Zaitsev's boundary conditions [2]. The spin-flip processes at the ferromagnetic layer are taken into account by the boundary conditions of Tokuyasu et al. [3] where a phenomenological parameter $\theta$ describes the degree of spin rotation. In this paper, we use the boundary condition in terms of the Maki-Schopohl parameterization of the Green's function $[4,5]$.

\section{Results}

We calculated the DOS in the normal metal at $x=0$ for $d_{n}=1.1 \xi_{0}$ for ideal $\left(\mathcal{T}_{0}=1\right.$, see Fig. 2$)$ and reduced transparency $\left(\mathcal{T}_{0}=0.8\right.$, see Fig. 3$)$ of the NS contact. We compared the case of a spin-active surface $(\theta=\pi / 4)$ with the spin-conserving case $(\theta=0)$ for various impurity concentrations. The order parameter was determined self-consistently at $T=0.1 T_{\mathrm{c}}$. In the dirty limit with a spin-conserving surface a mini gap can be observed; the DOS is almost unaffected when varying the transparency. Otherwise in the clean limit a clear dependence on the transparency occurs. Note that at a spin-active surface the mini gap is suppressed.

The sub-gap structure of the DOS in the normal metal stems from bound states due to Andreev reflection at the superconductor. In the clean case their energies can be calculated for a constant order parameter in the superconductor and ideal transparency at the NS contact with
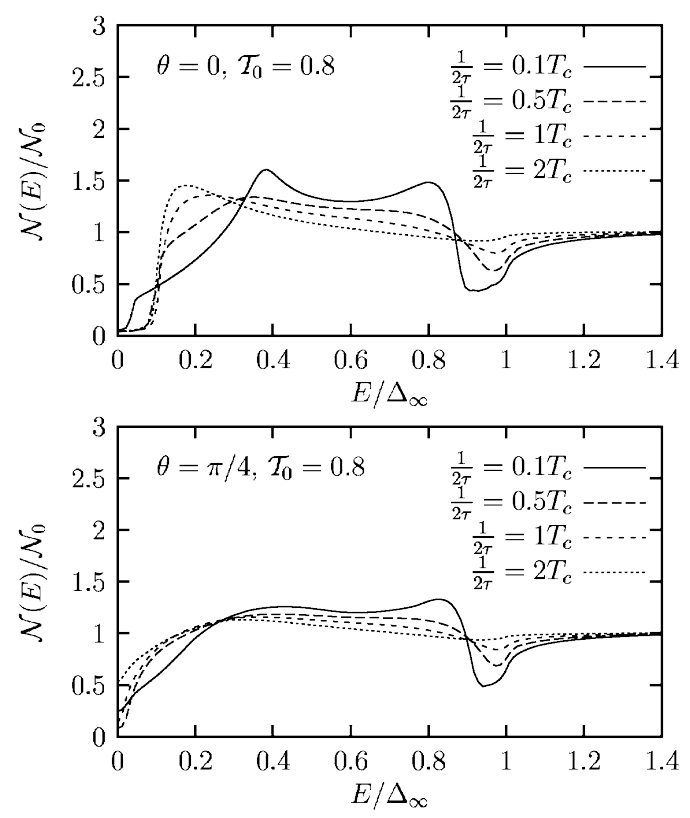

Fig. 3. For reduced transparency the mini gap is also suppressed in the magnetic case with increasing impurity scattering.

the result

$E^{2}=\frac{|\Delta|^{2}}{2}\left[1+\cos \theta \cos \frac{2 E L}{v_{F}} \pm \sin \theta \sin \frac{2 E L}{v_{F}}\right]$

with $L=2 d_{n} p_{F} / p_{F, x}$; the signs \pm describe the bound states in the two spin channels. In the non-magnetic case the low energy contribution to the DOS comes from trajectories with small angles of incidence $\left(L \gg d_{n}\right)$. These states are strongly affected by impurities in the normal metal. Their spectral weight is shifted to higher energies which results in a mini gap. For a magnetic surface the energies of these states are shifted up or down depending on the spin channel; this means that the spectral weight at low energies increases, and hence the mini gap is suppressed.

\section{Acknowledgements}

We thank M. Ozana and P. Schwab for fruitful discussions. T. Lück was supported by the Deutsche Forschungsgemeinschaft.

\section{References}

[1] W. Belzig, et al., Phys. Rev. B 54 (1996) 9443.

[2] A.V. Zaitsev, Sov. Phys. JETP 59 (1984) 1015.

[3] T. Tokuyasu, et al., Phys. Rev. B 38 (1988) 8823.

[4] A. Shelankov, M. Ozana, Phys. Rev. B 61 (2000) 7077.

[5] M. Eschrig, Phys. Rev. B 61 (2000) 9061. 